\title{
The Thompson effect is a function of the presence or absence of preoperative memories
}

\author{
DAVID J. BODART, MARY G. HATA, \\ DONALD R. MEYER, and PATRICIA M. MEYER \\ The Ohio State University, Columbus, Ohio 43212
}

\begin{abstract}
Rats were first prepared with unilateral injuries to the posterior cerebral cortex. Then they were trained on the black-white discrimination problem, and afterwards were tested for performance of the problem after having been prepared with contralateral posterior injuries. The animals exhibited complete forgetting of the habit. The finding indicates that the protection of retention that is commonly observed in subjects that are trained between two successive posterior ablations is a function of their having a preoperative memory to recall. It also contradicts predictions from the theory of cortical suppression and the theory of state-dependency.
\end{abstract}

It is thoroughly established that if normal rats are trained on a black-white discrimination problem, and are tested for postoperative performance of the problem after having been prepared with one-stage bilateral ablations of the visual neocortex, they exhibit an essentially complete forgetting of the problem but can nonetheless relearn it (Glendenning, 1972; Horel, Bettinger, Royce, \& Meyer, 1966; Lashley, 1935; Meyer, Yutzey, Dalby, \& Meyer, 1968; Meyer, Yutzey, \& Meyer, 1966). The phenomenon is interesting because, with other methods, it has also been shown that the injuries to the cortex do not destroy the engrams which were formed when the animals were trained before the operations (Braun, Meyer, \& Meyer, 1966; Jonason, Lauber, Robbins, Meyer, \& Meyer, 1970; LeVere \& Davis, 1977; LeVere \& Morlock, 1973; for review, cf. Meyer \& Meyer, 1977; or Meyer \& Meyer, in press).

The problems thus presented are as follows. Why should the subjects, if their memories are not much affected (if at all) by their injuries, be virtually unable to remember what they learned before the injuries were inflicted? In general, preoperative overtraining will not correct the animals' impairments (Glendenning, 1972; Lashley, 1921) although, as has recently been shown by Gray (1979), the deficit is partially reduced if the subjects are given extensive overtraining. However, if the animals are trained on the habit, and are then retrained after having been subjected to partial posterior injuries, a relatively small amount of retraining will convey a protection of performance of the problem following a second-stage ablation that completes the destruction of the posterior cortex (Glendenning, 1972; Spear and Barbas, 1975; Thompson, 1960). The

This research was supported, in part, by Grant MH-06211 from the National Institute of Mental Health to Donald R. Meyer and Patricia M. Meyer. retraining is essential because, as has been shown by Kircher, Braun, Meyer, and Meyer (1970), one-stage and two-stage posterior ablations have the same effects upon postoperative performance if serial preparations are not given interoperative training on the task.

There are several conceivable interpretations of the fact that postoperative forgetting is reduced if animals with partial posterior injuries are given retraining on the task. One was a hypothesis of Horel et al. (1966), which was based upon the fact that, ceteris paribus, normal rats can learn the black-white problem at a somewhat faster rate than animals with posterior injuries. Thus, they presumed that normal rats employ their cortical systems in preference to the systems that are utilized by animals with cerebral injuries for relearning of the problem after surgery because the former systems permit more efficient learning and performance of the task. However, that hypothesis was tested by Glendenning (1972), and was found by him to be inadequate because rats with unilateral posterior injuries that are retrained with occlusion of the ipsilateral eye are very slow to relearn the problem, but nonetheless exhibit partial protection of retention following a second-stage ablation that completes the destruction of the visual neocortex.

On those grounds, and others, Glendenning (1972) proposed that cortical systems, if intact, actively suppress the development, through practice, of subisocortical systems for remembering of the black-white problem. However, although the theory of suppression weathered several very stringent tests, the tests involved studies of posterior subjects that relearned with one eye occluded. The tests were subsequently shown to be invalid because such subjects, for some unknown reason, exhibit quick forgetting of the problem (cf. Meyer \& Meyer, 1977, for review).

A third interpretation of the fact that retraining following an injury to the posterior cortex conveys 
more protection than preoperative overtraining is provided by the notion that remembering is sometimes state-dependent. To the best of our knowledge, the concept has never been explicitly employed for such a purpose. However, the argument is reasonably straightforward, and as follows. If normal rats are trained on the black-white problem and are then prepared with posterior injuries, the posterior injuries induce a change of state and the animals are thereby prevented from remembering what they learned before the operations were performed. Hence, although the subjects still have a memory for the problem, the memory has been rendered unavailable and the animals can only recover the performance by learning a substitutive habit.

In the present experiment, the theory of suppression and the theory of state-dependency were tested through the use of comparatively simple procedures. Rats were first prepared with unilateral posterior injuries, then given training on the black-white problem, and were finally tested for performance of the problem after having been prepared with contralateral posterior or contralateral anterior injuries. The latter subjects served as the control group. The theory of suppression predicted that posterior-posterior subjects would relearn the problem with considerable savings on the grounds that the first-stage partial injuries would result in a partial release of suppression prior to training on the problem. The theory of state-dependency predicted that those preparations would relearn the problem with considerable savings on the grounds that a partial posterior injury would result in a partial induction of the state that is produced when the posterior cortex is completely destroyed. The experiment showed that neither prediction was correct, for the posterior-posterior animals exhibited complete forgetting of the black-white problem following their second operations.

\section{METHOD}

\section{Subjects}

The study was conducted with male Long-Evans rats, 90-120 days old at the beginning of training. They were randomly divided into two groups, 19 in a group designated as the P-A group, and 17 in a group designated as P-P. All animals were housed individually and maintained on an ad lib food and water schedule.

\section{Procedure}

The rats were trained on the black-white problem in the Thompson-Bryant apparatus (1955) with the spaced-trial training procedure that was introduced by Glendenning (1972). All were initially subjected to a unilateral posterior decortication using the pia-stripping method of Woolsey (cf. Meyer \& Meyer, 1971). The point of demarcation between anterior and posterior ablations was determined by using bregma as the landmark. Beginning on the fourth day postoperatively, and continuing through Day 9, each of the rats was handled for 5 min per day. On Day 10, each animal was allowed $15 \mathrm{~min}$ of free access to all areas of the training apparatus as an adaptive procedure. On Day 11 , as a pretraining technique, each rat was trained to run into the goal box to avoid foot shocks. Beginning on Day 12, each animal received initial training on the black-white discrimination problem until it reached a criterion of 9 correct responses in 10 trials. There was a limit of 25 trials per day, with short rests following the 8th and 16th trials. The rats were then subjected to either a completing unilateral posterior ablation or a contralateral anterior ablation. After an 11-day recovery period, the rats were retrained on the problem.

\section{Histology}

Following completion of retraining, the rats were given lethal doses of sodium pentobarbital. They were then perfused with normal saline followed by $10 \%$ Formalin. Diagrams of the extents of the surface lesions were made, and the brains frozen and sectioned at $30 \mu$. Every tenth section through the lesion and every fifth section through the thalamus were mounted and stained with cresyl violet. Subsequently, the dorsal lateral geniculate nuclei (LGNd) were studied to determine the extent of retrograde degeneration.

\section{RESULTS}

\section{Histological Results}

Figure 1 depicts the extents of the injuries to the

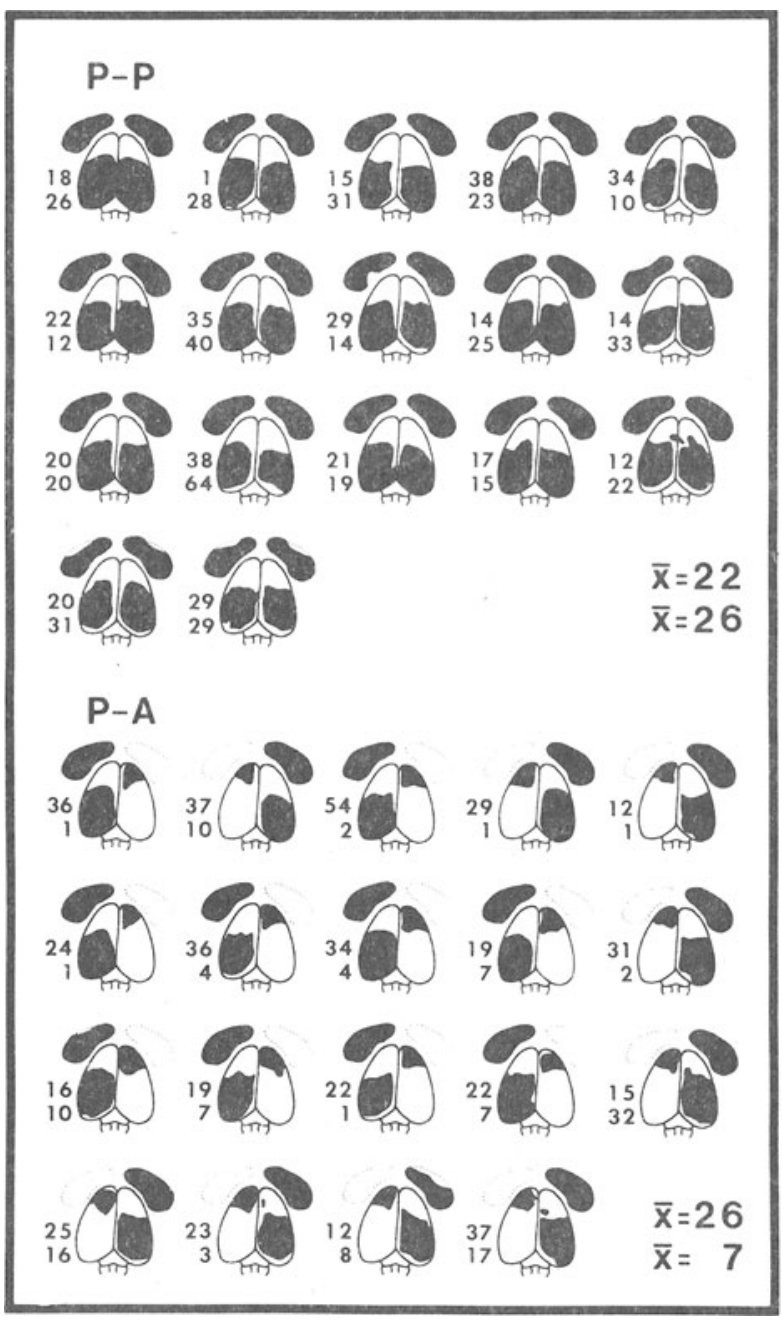

Figure 1. Surface Diagram and representation of degeneration in the LGNd's for Groups P-P and P-A. 
Table 1

\begin{tabular}{crr} 
& IOTR & \multicolumn{1}{c}{ RL } \\
\hline Group P-P & $22 \pm 2.5$ & $26 \pm 3.1$ \\
Group P-A & $26 \pm 2.5$ & $7 \pm 1.8$ \\
\hline
\end{tabular}

cortex and the extents of the retrograde degenerations of the LGNd's. The number of trials each rat required for interoperative learning and ultimate relearning is given beside each diagram. The first-stage posterior lesions of the two groups were very similar. The control anterior lesions were smaller than the secondstage posterior lesions, but were comparable in scope to the great majority of the anterior injuries of subjects that have previously been studied in our laboratory's program (cf. Meyer \& Meyer, 1977, for a discussion of the significance of variation in the scopes of anterior ablations).

\section{Behavioral Results}

Table 1 presents the interoperative learning and ultimate relearning scores for the two groups of subjects. The scores are the means, and standard errors of the means, for trials to criterion of $9 / 10$. The interoperative learning scores were not significantly different $(\mathrm{t}=1.22$; $\mathrm{df}=34)$, but the ultimate relearning score for Group P-P was significantly higher that the score for Group P-A $(\mathrm{t}=5.46 ; \mathrm{df}=34 ; \mathrm{p}<.001)$.

\section{DISCUSSION}

We propose to discuss the findings of this study in relation to the quantitative outcomes of several prior investigations. The data bank is summarized in Table 2 , in which the various experiments have been identified by the following codes: HBRM is Horel et al. (1966); KBMM is Kircher et al. (1970); GLEN is Glendenning 1972); ALEE is a study of Aileen Lee that is summarized by Meyer and Meyer (1977); GRAY is Gray (1979); BHMM is the present investigation; MYUM is Meyer et al. (1966); and MYDM is Meyer et al. (1968). Some of the groups were previously numbered for inclusion in a similar retrospective table by Gray (1979), and those groups are given the same completely arbitrary numbers.

PRE-1 and PRE-2 designate preoperative treatments. Thus, if a PRE-1 entry is BW25, it means that the subjects were trained on the black-white problem prior to surgery and reached a criterion of $9 / 10$ in approximately 25 spaced trials. If a PRE-2 entry is BW75, it means that the subjects were given an average of $75 \mathrm{BW}$ overtraining trials after having met criterion in PRE-1. SURG-1 and SURG-2 describe the surgical procedures; UVC is unilateral ablation of the visual cortex; UAC is unilateral ablation of the anterior cortex; and CVC is bilateral ablation of the posterior cortex.

IOTR-1 and IOTR-2 describe the interoperative training procedures. The IOTR-1 scores are the trials that were required for the subjects to reach the $9 / 10$ criterion; if the subjects were then given overtraining between operations, the numbers of overtraining trials are presented in IOTR-2. The POST-1 scores are the trials that were required for the subjects to relearn the black-white problem in their final postoperative tests of performance of the problem. When a given kind of treatment was omitted, there is no entry in the table; thus, two blank entries under PRE-1 and PRE-2 mean that the subjects received no training prior to their first operations.

Most of the scores were collected in experiments conducted with the spaced-trial training procedure that was used in this investigation. The exceptions were studies, marked with asterisks, in which a massed-trial procedure was employed. The scores for those groups are estimated spaced-trial scores that were obtained by dividing the massed-trial scores by 2 . The rule that was employed in the latter estimations was first suggested by Glendenning (1972), and its validity has since been supported by experiments of Howarth, Meyer, and Meyer (1979) and of Gray (1979). Thus, for example, the scores for the first group of subjects in the table, 2-HBRM, are estimated spaced-trial scores, while the scores for the second group of subjects in the table, 6-GLEN, are scores for

Table 2

\begin{tabular}{|c|c|c|c|c|c|c|c|c|}
\hline Study & $\mathrm{N}$ & PRE-1 & PRE-2 & SURG-1 & IOTR-1 & IOTR-2 & SURG-2 & POST-1 \\
\hline 2-HBRM* & 33 & BW25 & & CVC & & & & BW26 \\
\hline 6-GLEN & 14 & BW25 & & CVC & & & & BW23 \\
\hline 7-GLEN & 15 & BW27 & BW31 & CVC & & & & BW27 \\
\hline 15-GRAY & 10 & BW22 & BW78 & CVC & & & & BW14 \\
\hline 9-GLEN & 16 & BW25 & & UVC & BW10 & BW21 & UVC & BW13 \\
\hline 10-ALEE & 14 & BW25 & & UVC & BW08 & BW33 & UVC & BW07 \\
\hline 21-GRAY & 10 & BW25 & BW75 & UVC & BW04 & BW96 & UVC & BW06 \\
\hline 8-KBMM* & 79 & BW23 & & UVC & & & UVC & BW22 \\
\hline 1-BHMM & 17 & & & UVC & BW22 & & UVC & BW26 \\
\hline 2-BHMM & 19 & & & UVC & BW26 & & UAC & BW07 \\
\hline 3-MUYM* & 23 & BW27 & & CVC & & & & BW24 \\
\hline 4-MYDM* & 41 & BW26 & & CVC & & & & BW24 \\
\hline 5-KBMM* & 10 & BW24 & & CVC & & & & BW24 \\
\hline
\end{tabular}

*Studies where massed-trial procedure was employed and data have been transformed by a factor of one-half. 
comparably treated animals that were trained with the spaced-trial procedure.

Experiments 6-GLEN and 7-GLEN confirmed the classical conclusion of Lashley (1921) that reasonably extensive preoperative overtraining on the problem conveys no protection of performance of the task by CVC preparations. In 15-GRAY, it was shown that the conclusion was invalid for subjects with enormous amounts of overtraining, but the amount of protection observed in the study was only about 10 spaced trials. In 9-GLEN, which served to confirm, for our conditions, the validity of Thompson's (1960) effect, 31 trials of interoperative training produced about the same amount of protection as that which was observed in 15-GRAY for subjects that were given an average of 78 preoperative overtraining trials. In 10-ALEE, it was found that an additional 10 trials of IOTR would reduce the POST-1 score to 7 trials, or within 1 trial of the POST-1 score of subjects which, in 21-GRAY, were given 100 trials of preoperative training and then 100 trials of interoperative training.

From the foregoing findings, it is clear that training that is given to a UVC subject conveys more protection of performance of the problem than preoperative overtraining. Also, and whatever the reason for the difference, it has nothing to do with the fact that the subjects that were given interoperative training were prepared with UVC-UVC ablations, while the subjects that were given overtraining prior to surgery were prepared with CVC ablations. Thus, as was shown in 8-KBMM, UVC-UVC subjects have the same impairments of POST-1 performance of the problem as CVC subjects, if IOTR is omitted (cf. POST-1 8-KBMM with POST-1 2-HBRM, 6-GLEN, 3-MYUM, 4-MYDM, and 5-KBMM).

The principal finding of this investigation is that IOTR is ineffective if given to UVC-UVC subjects that have had no preoperative BW training. Thus, Group 1-BHMM, after having learned the problem interoperatively in 22 trials, required 26 trials to relearn the problem following their second-stage ablations. Hence, their performance was not only substantially worse than the performance of the UVC-UAC subjects of 2-BHMM, but was also no better than that of the 8-KBMM subjects that received no training between serial UVC-UVC ablations. Moreover, the score was approximately the same as the actual or estimated scores for the CVC subjects of 2-HBRM, 6-GLEN, 3-MYUM, 4-MYDM, and 5-KBMM.

As we have observed, the findings contradict predictions from the theory of cortical suppression and the theory of state-dependency. Also, they are moderately at odds with a finding of Thompson (1960), who observed that serial UVC-UVC subjects that were not trained prior to the first operation exhibited a partial protection of retention when given interoper- ative training (his Group UVLR). Our own observations suggest that his effect is a function of preoperative training, and we are reasonably sure that those results are accurate because a replication of 1-BHMM has since been performed in our laboratory (unpublished observations of Robert Dewberry; $\mathrm{n}=12$; IOTR $-1=26 \pm 2.7$ spaced trials; POST $-1=21 \pm 3.0$ spaced trials).

In closing, we will note the findings compromise a term in the rule of maintenance of access that has been proposed by Meyer and Meyer (1977). According to the rule, protection of postoperative performance of the black-white problem can only be obtained by retraining a subject that has first sustained a posterior injury. The term in question was based upon the fact that subjects that are trained on the task after having been prepared with bilateral ablations of the anterior cortex exhibit no protection from the deficits produced by second-stage posterior ablations (Horel et al., 1966). That finding was interpreted by Meyer and Meyer to mean that the Thompson (1960) effect is conditional upon a preexisting visual injury, but the present observations suggest that the result was due to the fact that the subjects had received no training on the task before their first-stage anterior ablations. But whether that is so or not remains to be decided, and we think that the question is important.

\section{REFERENCES}

Braun, J. J., Meyer, P. M., \& Meyer, D. R. Sparing of a brightness habit in rats following visual decortication. Journal of Comparative \& Physiological Psychology, 1966, 61, 79-82.

Glendenning, R. L. Effects of training between two unilateral lesions of visual cortex upon ultimate retention of black-white habits by rats. Journal of Comparative \& Physiological Psychology, 1972, 80, 216-229.

GrAY, T. S. Facilitory effects of multiple task training and overtraining upon the ultimate retention of visual discriminations in rats with bilateral ablations of the posterior neocortex. Unpublished doctoral dissertation at The Ohio State University, 1979.

Horel, J. A., Bettinger, L. A., Royce, G. J. \& Meyer, D. R. Role of neocortex in the learning and relearning of two visual habits by the rat. Journal of Comparative \& Physiological Psychology, 1966, 61, 66-78.

Howarth, H. H., Meyer, D. R., \& Meyer, P. M. Perinatal injuries to the visual cortex enhance the significance of extravisual cortex for performance of a visual habit. Physiological Psychology, 1979, 7, 163-166.

Jonason, K. R., Lauber, S. M., Robbins, M. J., Meyer, P. M. \& MEYER, D. R. Effects of amphetamine upon relearning pattern and black-white discriminations following neocortical lesions in rats. Journal of Comparative \& Physiological Psychology, 1970, 73, 47-55.

Kircher, K. A., Braun, J. J., Meyer, D. R. \& Meyer, P. M. Equivalence of simultaneous and successive neocortical ablations in production of impairments of retention of black-white habits in rats. Journal of Comparative \& Physiological Psychology, 1970, 71, 420-425.

LASHLEY, K. S. Studies of cerebral function in learning. II. The effects of long-continued practice upon cerebral localization. Journal of Comparative Psychology, 1921, 1, 453-468. 
LAShley, K. S. The mechanism of vision: XII. Nervous structures concerned in habits based on reactions to light. Comparative Psychology Monographs, 1935, 11, 43-79.

LEvere, T. E., \& DAvis, N. Recovery of function after brain damage: The motivational specificity of spared neural traces. Experimental Neurology, 1977, 57, 883-889.

LeVere, T. E., \& MorLock, G. W. Nature of visual recovery following posterior neodecortication in the hooded rat. Journal of Comparative \& Physiological Psychology, 1973, 83, 62-67.

Meyer, D. R., \& Meyer, P. M. Dynamics and bases of recoveries after injuries to the cerebral cortex. Physiological Psychology, 1977, 5, 133-165.

Meyer, D. R., \& MEYer, P. M. Inductions of recoveries from amnesias. In J. L. McGaugh \& R. F. Thompson (Eds.), Learning and memory. New York: Plenum, in press.

Meyer, D. R., Yutzey, D. A., \& Meyer, P. M. Effects of neocortical ablations on relearning of black-white discrimination habit by two strains of rats. Journal of Comparative \& Physiological Psychology, 1966, 61, 83-86.
Meyer, P. M., Yutzey, D. A., Dalby, D. A., \& Meyer, D. R. Effects of simultaneous septal-visual, septal-anterior and anterior-posterior lesions upon relearning a black-white discrimination. Brain Research, 1968, 8, 281-290.

Spear, P. D., \& Barbas, H. Recovery of pattern discrimination ability in rats receiving serial or one-stage visual cortex lesions. Brain Research, 1975, 94, 337-346.

Tном PSON, R. Retention of a brightness discrimination following neocortical damage in the rat. Journal of Comparative \& Physiological Psychology, 1960, 53, 212-215.

Thompson, R., \& BRYANT, J. H. Memory as affected by activity of the relevant receptor. Psychological Reports, 1955, 1, 393400 .

(Received for publication December 4, 1979; accepted December 20,1979.) 